$11,16,19$

\title{
Теплота плавления малых кластеров в модели потенциала с эффективной глубиной потенциальной ямы
}

\section{(C) Г.А. Мельников}

Юго-Западный государственный университет (ЮЗГУ), Курск, Россия

E-mail: melnikovga@mail.ru

(Поступила в Редакцию 25 октября 2017 г. В окончательной редакции 27 ноября 2017 г.)

В рамках кластерной модели с использованием парного степенного потенциала взаимодействия Ми с эффективной глубиной потенциальной ямы, глубина которой определяется числом частиц в кластере, получено соотношение для расчета теплоты плавления кластерной системы. Полученное математическое соотношение не содержит эмпирических постоянных и показывает, что отношение теплоты плавления кластера к теплоте плавления макроскопического образца является универсальной функцией числа частиц в кластере и математически описывается квадратом гиперболического тангенса.

DOI: 10.21883/FTT.2018.05.45800.304

\section{1. Введение}

Физико-химические свойства кластерных систем и наночастиц, в том числе температура и теплота плавления, подвержены влиянию размерных эффектов. Такие эффекты в малых кластерных системах (РЭМКС) проявляются особенно ярко [1-10]. Для температуры плавления предложено более десятка формул, отображающих зависимость этой величины от размеров (обычно радиуса или диаметра наночастицы), в конечном счете они являются модификациями формулы Томсона $[4,5]$. В настоящее время фазовые переходы в кластерных системах исследуются теоретически и экспериментально довольно интенсивно. Следует отметить подробное изучение процессов плавления атомных и молекулярных кластеров в работах Смирнова [1-4], Берри (Berry) с сотрудниками [5-10], металлических кластеров в работах Ределя, Гафнера, Гафнера и др. [11-13], металлических и молекулярных кластеров Егорова, Урюпина, Иванова и др. $[14,15]$. В обзорной статье Макарова приводится обширный список публикаций по проблемам описания процессов плавления в кластерных системах и наночастицах [16]. Описание различных направлений в исследовании размерных эффектов в кластерных системах можно найти в цитируемой литературе работ [1-15]. В данной статье предложен метод расчета температуры и теплоты плавления малых кластеров на основе степенного потенциала взаимодействия между частицами с эффективной глубиной потенциальной ямы, зависящей от числа частиц в кластерной системе, применение которого позволило установить зависимость температуры и теплоты плавления кластера от числа частиц в его структуре.

\section{2. Потенциал взаимодействия Ми с эффективной глубиной потенциальной ямы}

Исследование кластерных систем одноатомных и органических веществ показало, что взаимодействие между частицами кластерной системы можно успешно описать парным степенным потенциалом Ми, однако недостаточно правильно выбрать значения параметров $m, n$, $\varepsilon_{0}$ и $\sigma_{0}$ в этом потенциале взаимодействия. Необходимо предположить, что глубина потенциальной ямы в потенциале зависит от числа частиц в кластерной системе и параметров состояния среды. В таком случае глубина потенциальной ямы является эффективной глубиной $\varepsilon_{\text {eff }}$ и является функцией числа частиц в системе, плотности и температуры. К аналогичному выводу пришли авторы работ $[17,18]$.

Степенной потенциал взаимодействия Густава Ми записывается в виде [19]

$$
\varphi\left(r_{i j}\right)=\frac{\varepsilon_{0}}{n-m}\left(\frac{n^{n}}{m^{m}}\right)^{\frac{1}{n-m}}\left[\left(\frac{\sigma_{0}}{r_{i j}}\right)^{n}-\left(\frac{\sigma_{0}}{r_{i j}}\right)^{m}\right]
$$

где $\varepsilon_{0}-$ глубина потенциальной ямы, $\sigma_{0}-$ эффективный диаметр взаимодействующих частиц, $n \geq m-$ целочисленные показатели степени, причем $m=6, n=9-24$.

В предложенной авторами модели классический степенной потенциал Ми (1) необходимо изменить, заменив величину $\varepsilon_{0}$ на эффективную глубину потенциальной ямы [20]

$$
\varepsilon_{\mathrm{eff}}=\varepsilon_{0}\left[1+\left(\frac{Z-1}{Z+1}\right)^{2}\left(\frac{k T}{\varepsilon_{0}}\right)\right]
$$


тогда модернизированный степенной парный потенциал Ми запишется в виде

$$
\begin{gathered}
\varphi\left(r_{i j}\right)=C_{i j} \varepsilon_{0}\left[1+\left(\frac{Z-1}{Z+1}\right)^{2} \frac{k T}{\varepsilon_{0}}\right]\left[\left(\frac{\sigma_{0}}{r_{i j}}\right)^{n}-\left(\frac{\sigma_{0}}{r_{i j}}\right)^{m}\right], \\
C_{i j}=\frac{n}{(n-m)}\left(\frac{n}{m}\right)^{\frac{m}{n-m}}, \quad \sigma_{0}=\left(\frac{m}{n}\right)^{\frac{1}{n-m}} R_{1}
\end{gathered}
$$

где $Z$ - число частиц в кластерной системе.

Для макроскопических образцов установлено эмпирическое правило, согласно которому температура плавления вещества пропорциональна глубине потенциальной ямы парного потенциала взаимодействия [1-4]. В первом приближении это правило выполняется для кластерных систем и наночастиц [21]. С условием модернизации потенциала Ми для кластерных систем можно предположить, что температура плавления такой системы пропорциональна эффективной глубине потенциальной ямы потенциала взаимодействия: $T_{m}(Z)=c \varepsilon_{\text {eff, }} c=$ const.

Эмпирическая постоянная $c$ определится по предельному переходу. Для макроскопического образца при $Z \rightarrow \infty, \quad T_{m}(Z) \rightarrow T_{m}(\infty)$, т. е. температура плавления кластерной системы стремится к температуре плавления макроскопического образца. Для эмпирической постоянной получено значение

$$
c=\frac{2}{3}(\Phi-1)=0.412 \ldots
$$

С учетом найденного значения эмпирической постоянной (4) соотношение для расчета температуры плавления кластера, содержащего $Z$ частиц, запишется в виде формулы

$$
T_{m}(Z)=0.56(56) \ldots(\Phi-1) \varepsilon_{0}\left[1+\left(\frac{Z-1}{Z+1}\right)^{2}\right],
$$

где $\Phi=1.6180339 \ldots$ - „золотое“ сечение, которое определяет критерий Линдемана при плавлении кластерных систем [22].

Глубина потенциальной ямы парного потенциала взаимодействия в формуле (5) зависит от вида выбранного потенциала или определяется на основе эмпирических соотношений, поэтому прогнозирование температуры плавления кластерных систем по этой формуле обладает неопределенностью.

Формулу (5) удобно записать так

$$
T_{m}(Z)=\frac{1}{2} T_{m}(\infty)\left[1+\left(\frac{Z-1}{Z+1}\right)^{2}\right],
$$

где $T_{m}(\infty)$ - температура плавления соответствующего объемного образца, которую можно определить экспериментально с хорошей точностью.

Для кластерных систем с конечным числом частиц температура плавления, приведенная к температуре плавления соответствующего макроскопического образца, является универсальной функцией числа частиц в их структуре.

Используя определение гиперболического тангенса заметим, что его квадрат совпадает с поправкой на учет много частичного взаимодействия в формуле для глубины эффективной потенциальной ямы (2), поэтому запишем

$$
\tanh ^{2} x=\left[\frac{\exp (2 x)-1}{\exp (2 x)+1}\right]^{2}=\left(\frac{Z-1}{Z+1}\right)^{2} .
$$

Если под числом частиц в структуре кластера понимаем величину $Z=\exp (2 x)$, то получим универсальную гиперболическую функцию

$$
\frac{2 T_{m}(x)}{T_{m}(\infty)}=1+\tanh ^{2} x
$$

Полученное соотношение для расчета температуры плавления кластерных систем (8) не содержит эмпирических постоянных, удовлетворяет предельным переходам и согласуется с экспериментальными данными, полученными различными методами.

\section{3. Теплота плавления малых кластерных систем}

Для макроскопических образцов их теплота плавления пропорциональна температуре плавления, по крайней мере это хорошо выполняется для кристаллов благородных газов, органических веществ и металлов. Зависимость теплоты плавления нанокластеров от их размера для золота, меди, алюминия и кобальта подробно проведено в работе [23] путем моделирования методом Монте-Карло с использованием многочастичного потенциала Гупта и показано, что зависимости температуры $T_{m}(R)$ и теплоты плавления $\Delta H_{m}(R)$ близки к линейным в координатах $T_{m}(R), \Delta H_{m}(R)=f\left[R^{-1}, Z^{1 / 3}\right]$.

В первом приближении размерные зависимости для теплоты плавления и температуры плавления для нанокластеров должны совпадать по функциональному виду [23]. Это подтверждается в рамках приближения „скин-слоя“ для размерного эффекта, в котором зависимость теплоты плавления от размеров кластера определяется формулой [24]

$$
\frac{\Delta H_{m}(R)}{\Delta H_{m}(\infty)}=\left[1-\left((R+\delta) / R_{0}-1\right)^{-1}\right]
$$

где $\delta$ - величина скин-слоя, $R_{0}=(3-d) a-$ характерный размер объекта, $d, a-$ размерность и эффективный диаметр объекта.

Опираясь на положение о тождественной функциональной зависимости температуры и теплоты плавления кластерных систем от их размера и численного состава, 
Теплота плавления кластера $Z(13)$ для некоторых веществ

\begin{tabular}{|c|c|c|c|c|c|c|c|c|}
\hline \multirow[t]{2}{*}{ № } & \multirow[t]{2}{*}{ Substance } & \multicolumn{2}{|c|}{$\Delta H_{m}(\infty)$} & \multicolumn{2}{|c|}{$\begin{array}{c}\text { Потенциал } \\
\text { Леннард-Джонса }[26,27]\end{array}$} & \multicolumn{3}{|c|}{$\Delta H_{m}(13)$} \\
\hline & & $\mathrm{J} / \mathrm{mole}$ & $\mathrm{K}$ & $\sigma_{0}, \mathrm{~A}$ & $\varepsilon_{0} / k, \mathrm{~K}$ & $\mathrm{~J} / \mathrm{mole}$ & $\mathrm{K}$ & $\Delta H_{m}(13) / \varepsilon_{0}$ \\
\hline 1 & Neon [27] & 335 & 40.3 & $\begin{array}{l}3.090 \\
2.775 \\
2.91^{(*)}\end{array}$ & $\begin{array}{l}35.6 \\
36.8[30] \\
44.3\end{array}$ & 290.5 & 35.0 & $\begin{array}{l}\mathbf{1 . 0 0} \\
0.95 \\
0.79\end{array}$ \\
\hline 2 & Argon [27] & 1177 & 141.7 & $\begin{array}{l}3.405 \\
3.401 \\
4.05\end{array}$ & $\begin{array}{l}119.8 \\
116.8[30] \\
122.5\end{array}$ & 1021 & 122 & $\begin{array}{l}\mathbf{1 . 0 2} \\
1.04 \\
1.00\end{array}$ \\
\hline 3 & Krypton [27] & 1638 & 197.1 & $\begin{array}{l}3.600 \\
3.601 \\
4.12\end{array}$ & $\begin{array}{l}171.0 \\
164.6[30] \\
188.8\end{array}$ & 1232 & 148 & $\begin{array}{l}\mathbf{0 . 8 7} \\
0.90 \\
0.78\end{array}$ \\
\hline 4 & Xenon [27] & 2900 & 276.8 & $\begin{array}{l}4.020 \\
4.055 \\
4.79\end{array}$ & $\begin{array}{l}243.4 \\
218.2[30] \\
225.9\end{array}$ & 2515 & 303 & $\begin{array}{l}\mathbf{1 . 2 4} \\
1.39 \\
1.34\end{array}$ \\
\hline 5 & Oxygen [27] & 445 & 53.5 & $\begin{array}{l}3.880 \\
3.72\end{array}$ & $\begin{array}{l}118.0 \\
158.6\end{array}$ & 335 & 40.3 & $\begin{array}{l}\mathbf{0 . 3 4} \\
0.25\end{array}$ \\
\hline 6 & Nitrogen [27] & 720 & 86.6 & $\begin{array}{l}3.613 \\
4.42\end{array}$ & $\begin{array}{r}103.8 \\
96.0\end{array}$ & 624 & 75.1 & $\begin{array}{l}\mathbf{0 . 7 3} \\
0.78\end{array}$ \\
\hline 7 & Benzene [28] & 9910 & 1193 & $\begin{array}{l}5.26 \\
2.926\end{array}$ & $\begin{array}{l}531 \\
888[29]\end{array}$ & 8594 & 1034 & $\begin{array}{l}\mathbf{1 . 9 5} \\
1.16\end{array}$ \\
\hline 8 & Toluene $[28]$ & 6633 & 798 & 5.64 & 575 & 5753 & 692 & 1.20 \\
\hline
\end{tabular}

(*) Примечание. Третья строка в разделе потенциал Леннард-Джонса относится к параметрам потенциала Морзе [27], для бензола - вторая строка к параметрам потенциала Кихары [29].

на основе соотношения (8) для теплоты плавления получим формулу

$$
\frac{\Delta H_{m}(x)}{\Delta H_{m}(\infty)}=\frac{1}{2}\left(1+\tanh ^{2} x\right)
$$

где $x=\frac{1}{2} \ln (Z)$ - параметр плавления, определяющий теплоту плавления кластерной системы, содержащей $Z$ частиц.

Таким образом, согласно полученному соотношению (10) теплота плавления кластерной системы определяется числом частиц в такой системе и свойствами гиперболического тангенса. В частности для кластера из трех частиц $(Z=3)$, т. е. тримера вещества, для теплоты плавления тримера получим

$$
\Delta H_{m}(Z=3)=\frac{5}{8} \Delta H_{m}(\infty) \approx \frac{1}{\Phi} \Delta H_{m}(\infty) .
$$

Теплота плавления макроскопических кристаллов $\Delta H_{m}(\infty)$ определяется экспериментально с хорошей точность, что позволяет оценить теплоту плавления тримера вещества $\Delta H_{m}(Z=3)$ согласно формуле (11) так же с приемлемой точностью и сравнить с результатами машинного моделирования [25].

В таблице приведены результаты расчета теплоты плавления для кластеров благородных газов и некоторых углеводородов. В стандартных теплофизических измерениях можно зафиксировать изменение температуры объекта порядка $0.05^{\circ}$, следовательно согласно формуле (6) для кластеров аргона (температура плавления $83.78 \mathrm{~K}$ ) размерный эффект по температуре можно зафиксировать экспериментально в кластерах, содержащих порядка 3000 атомов. Радиус кластера составляет порядка $20 \mathrm{~nm}$, что в настоящее время является пределом в получении наночастиц такого размера.

Анализ таблицы показывает, что теплота плавления кластера с числом частиц $Z=13$, отнесенная к глубине потенциальной ямы потенциала Леннард-Джонса для благородных газов лежит в пределах 0.87-1.24, однако параметры потенциала Леннард-Джонса, полученные различными методами, значительно разнятся. В работе [26] приводятся данные для параметров потенциала Леннард-Джонса для благородных газов, метана и азота, которые показывают, что различные источники приводят значения для параметров потенциала с разбросом в десятки процентов. Например, для криптона глубина потенциальной ямы принимает значения от $158.0 \mathrm{~K}$ до $191.4 \mathrm{~K}$ (различие составляет порядка 20\%), для аргона это различие еще больше от $93.3 \mathrm{~K}$ до $141.2 \mathrm{~K}$ (различие составляет порядка 50\%), поэтому отношение $\Delta H_{m}(13) / \varepsilon_{0}$ можно принять равным единице с погрешностью порядка $20 \%$. Одной из особенностей кластера с числом частиц $Z=13$ заключается в том, что теплота плавления такого кластера равна глубине потенциальной ямы парного степенного потенциала взаимодействия для благородных газов.

В работе [2] приводятся данные для энергий нижних конфигурационных состояний леннард-джонсовского кластера с $Z=13$, из которых следует, что переход кластера из твердого состояния в жидкое сопровождается выделением энергии $\Delta H_{m}(13)=0.56-0.94$ в единицах 
глубины потенциальной ямы, что согласуется с представленными в работе вычислениями.

Для органических жидкостей применение потенциала Леннард-Джонса не совсем корректно, поэтому для бензола были взяты кроме параметров этого потенциала значение параметров потенциала Кихары [29], тогда $\Delta H_{m}(13) / \varepsilon_{\text {Kich. }}=1.16$ в единицах глубины потенциальной ямы потенциала Кихары.

Полученное соотношение (8) может служить независимым методом для оценке теплоты плавления кластерных систем различного состава.

\section{4. Заключение}

В литературе имеется обширный теоретический и экспериментальный материал по исследованию размерного эффекта для различных физических характеристик кластерных систем и наночастиц. Одним из важных выводов таких исследований является установление факта, что температура и теплота плавления кластеров определяется глубиной потенциальной ямы потенциала взаимодействия между частицами, поэтому остается важнейшей задачей физики кластерных систем поиск реальных потенциалов взаимодействия между одноатомными частицами и сложными молекулами. Применение сложных потенциалов, например потенциала сглаженных коллапсирующих сфер с отрицательной кривизной в области отталкивания объясняет существование фазовых переходов и описывает аномальное поведение кластерной системы, однако можно с успехом использовать более простые двухчастичные потенциалы с меняющейся глубиной потенциальной ямы.

Глубину потенциальной ямы парного потенциала взаимодействия необходимо считать функцией либо параметров состояния системы, либо функцией числа частиц в структуре кластеров. В этом случае температура и теплота плавления становится функцией числа частиц в кластерной системе. Удачно найденная функция, задающая эффективную глубину потенциальной ямы парного потенциала взаимодействия от числа частиц в кластере с учетом предельного перехода к макроскопическим образцам, позволяет получить универсальное математическое соотношение, не содержащее эмпирических постоянных и описывающее размерные эффекты в кластерных системах.

Сложная процедура описания взаимодействия между частицами в кластерах с помощью многочастичных потенциалов может быть заменена описанием посредством двухчастичного потенциала взаимодействия с эффективной глубиной потенциальной ямы. Наиболее общим двухчастичным степенным потенциалом взаимодействия является потенциал Ми, позволяющий так же варьировать показатель степени в отталкивающей части в широких пределах, поэтому применение модифицированного потенциала Ми для описания кластерных систем вполне оправдано.
Автор выражает благодарность Н.М. Игнатенко за полезные обсуждения материала и ценные замечания.

\section{Список литературы}

[1] Б.М. Смирнов. УФН 164, 11, 1165 (1994).

[2] Р.С. Берри, Б.М. Смирнов. УФН 175, 4, 367 (2005).

[3] Р.С. Берри, Б.М. Смирнов. УФН 179, 2, 147 (2009).

[4] Р.С. Берри, Б.М. Смирнов. УФН 183, 10, 1029 (2013).

[5] R.S. Berry, J. Jellinek, G. Natanson. Phys. Rev. A 30, 2, 919 (1984).

[6] R.S. Berry. Chem. Rev. 93, 7, 2379 (1993).

[7] R.S. Berry. Nature 393, 212 (1998).

[8] R.S. Berry. Theory of Atomic and Molecular Clusters. (Springer Berlin) (1999).

[9] R.S. Berry, B.M. Smirnov. ЖЭТФ 125, 2, 414 (2004).

[10] J. Jellinek, T.L. Beck, R.S. Berry. J. Chem. Phys. 84, 5, 2783 (1986).

[11] Л.В. Редель, Ю.Я. Гафнер, С.Л. Гафнер. ФТТ 57, 10, 2061 (2015).

[12] С.Л. Гафнер, Л.В. Редель, Ю.Я. Гафнер. ЖЭТФ 141, 3, 488 (2012).

[13] Ж.В. Головенько, С.Л. Гафнер, Л.В. Редель, Ю.Я. Гафнер. Изв. вузов. Физика 1-2, 178 (2011).

[14] В.М. Егоров, О.Н. Урюпин, Ю.В. Иванов. ФТТ 57, 9, 1798 (2015).

[15] В.М. Егоров, В.А. Марихин, Л.П. Мясникова. ФТТ 50, 1, 123 (2008).

[16] Г.Н. Макаров. УФН 180, 2, 185 (2010).

[17] Hong-Chul Park, Ki-Taek Byun, Ho-Young Kwak. Chem. Eng. Sci. 60, 7, 1809 (2005).

[18] A. Kh. Al-Matar, A.H. Tobgy, I.A. Sulieman. Mol. Simulation 34, 3, 289 (2008).

[19] G. Mie. Ann. Phys. 11, 8, 657 (1903).

[20] G.A. Melnikov. IOP Conf. Ser. Mater. Sci. Eng. 168, 012020 (2017).

[21] S.Yu. Davydov. Semicond. Phys. Technology 49, 1683 (2015).

[22] G.A. Melnikov, Н.М. Игнатенко, В.Г. Мельников, Е.Н. Черкасов, О.А. Манжос. Моделирование структур, строение вещества, нанотехнологии. Сб. материалов III Межд. научн. конф. Тула (2016). С. 49-54.

[23] Н.Ю. Сдобняков, П.В. Комаров, А.Ю. Колосов, Н.В. Новожилов, Д.Н. Соколов, Д.А. Кульпин. Конденсированные среды и межфазные границы 15, 3, 337 (2013).

[24] Q. Jiang, H.X. Shi, M. Zhao. J. Chem. Phys. 111, 5, 2176 (1999).

[25] D.J. Wales, J.P.K. Doye, A. Dullweber, M.P. Hodges, F.Y. Naumkin, F. Calvo, J. Hernández-Rojas, T.F. Middleton. The Cambridge Cluster Database (2012). http://www-wales.ch.cam.ac.uk/CCD.html

[26] F. Cuadros, I. Cachadina. Mol. Eng. 6, 319 (1996).

[27] A. Matsumoto. Verlag der Zeitschrift für Naturforschung, T'ubingen. http://znaturforsch.com (2011).

[28] G.J. Graziano. Phys. Chem. B 109, 2, 981 (2005).

[29] C. Vega, S. Lago, R. Pospisil, S. Labik, A. Malijevsky. J. Phys. Chem. 96, 1895 (1992).

[30] Oh. Seung-Kyo. J. Thermodynamics ID 828620, 29 (2013). 